\title{
PERFORMANCE IMPROVEMENT FOR A WET BENCH TOOL
}

\author{
Kamil Erkan Kabak \\ Cathal Heavey \\ Enterprise Research Centre \\ University of Limerick \\ Limerick, IRELAND
}

\author{
Vincent Corbett \\ Analog Devices \\ Limerick, IRELAND
}

\begin{abstract}
Cluster tools are prevalent in wafer fabs. The main reason for this prevalence is that the integration of simple sequential steps together with wafer handling equipment reduces the cost significantly with shared facitilies and smaller foot-prints. This paper analyzes the performance of a wet bench tool in a wet cleaning process by means of a detailed simulation model under different operating factors. The results of the simulation experiments show that through reconfiguration of the recipe sequence types that a $18 \%$ improvement in average hourly throughput can be realised under the same average cycle time.
\end{abstract}

\section{INTRODUCTION}

In wafer fabrication, wet bench tools are used for wet cleaning and etching processes. They play a crucial role in wafer fabrication since the overall cleaning process constitues about $10 \%$ of the operations in a wafer fab. The wet bench under examination has a large number of recipes with varying process times. The objective of this study is to minimize the average cycle time of the wet bench tool to improve the tool performance and to support more robust tool operation by evaluating varying operating scenarios with a detailed simulation model.

In this paper, first the wet bench tool under examination is briefly introduced. Then, the literature research is introduced. After that, the simulation model and the model inputs are explained briefly together with model outputs and simulation validation. Finally, the results of simulation experiments along with concluding remarks are presented at the end of paper.

\section{WET BENCH TOOL DESCRIPTION}

The wet bench tool of interest includes six etch and six rinse baths (i.e. chemical and water baths), an additional rinse tank to clean robot transfer arms, a dryer module and a shared transfer module between input and output as illustrated in Figure 1. Also, the tool has an auxiliary material handling robot which transfers the carrier from one tank to another tank according to the recipe of the lots in the carrier. That is, the lots having the same recipe in the carrier are transferred strictly from one tank to another by a bath sequence (i.e tank route) which is defined by the associated recipe (Lee, Lee, and Lee 2007). There is no intermediate buffer where partial processed batches or jobs can wait at the tool.

There are a number of operational constraints that complicates the operation of the tool. These constraints include collisions for robot and deadlocks. To illustrate, the robot will never deliver a carrier to a full tank since a bath can process only one batch at a time, also the robot will never transfer a carrier to a bath if parameters of the bath go beyond the limits specified by the recipe. Also there are strict no-wait (NW) or zero-wait (ZW) time constraints for the chemical or etch baths. In other words, a batch should be removed to its next rinse bath immediately after its chemical bath, since overprocessing at a chemical bath causes significant quality problems and overexposed batches 
Kabak, Heavey and Corbett

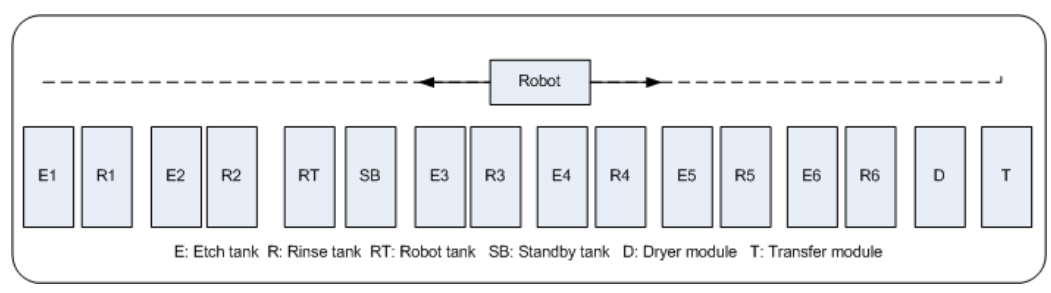

Figure 1: Schematic view of the wet bench tool.

are generally scrapped. On the other hand, overprocess at a rinse tank may not cause problems and generally batches can stay in deionized water. The next section surveys previous studies on wet benches and discusses the solutions for tool performance analysis.

\section{LITERATURE RESEARCH}

There is little research considering operational planning and control of the wet bench tool among the cluster tool types in semiconductor manufacturing. (Mauer, Schelasin, and Miller 1992) points out the non-linear relationship between the cycle time and the throughput in wet bench type of cluster tools. They analyze the cycle time-throughput relationship by increasing the number of carriers in the wet bench tool. (Geiger, Kempf, and Uzsoy 1997) define the wet bench operation as an $m$-machine permutation flow shop sequencing problem in order to minimize the makespan. Different from the study reported here, they assume the same bath sequence for all jobs in the tool. In other words, they use permutation schedules. In our study, the bath sequences are defined by recipes. Similar to (Geiger, Kempf, and Uzsoy 1997), Bhushan and Karimi (2003) analyze the problem as $m$-machine serial flow shop problem and they present a mixed-integer linear programming (MILP) formulation to minimize the makespan. Govind and Fronckowiak (2003) present a detailed description of a hybrid simulation model of wet benches by describing the wet bench operation through an event-graph. They examine average cycle time and tool set utilization for three scheduling policies by using a two-level full factorial experimental design. Quek et al. (2007) apply different dispatching rules and recipe dedication schemes on the performance of wet bench tools. They conclude that $8 \%$ of more capacity is obtained with more recipe variations at low loading levels. Finally, (Lee, Lee, and Lee 2007) present a wet bench model with multi-robot zones in a petri net application. They also solve the problem by using a mixed integer programming model with operational constraints.

\section{SIMULATION MODEL}

In this study, a wet bench tool in the wet cleaning process is examined by a detailed simulation model created by Siemens Tecnomatix Plant Simulation 8.2 Software (Siemens 2008). There are a number of assumptions considered for the analysis. These assumptions are: (1) Nonpreemptive process at a bath or robot tranfer is considered. (2) No random failures are assumed for robot and baths (3) No chemical change or setup time is considered when the recipe type is changed in the baths. (4) No preventive maintenance is assumed since chemical change is required after the tool has run for a pre-defined number of batches. Apart from assumptions, there are some operational constraints or critical rules defined for the tool operation, these constraints are:

- Each etch bath should be processed exactly with its recipe time, if the lots stays more than their recipe time in an etch bath then they will be scrapped,

- The rinse tank should be empty to start a process in an etch tank. That is, lots are not introduced into an etch tank until its rinse tank is free,

- The robot prioritizes removing lots from etch tanks,

- Dryer Time - it is the time a boat spends in the dryer, it is the same for all type of recipes like transfer time,

- Recipe Sequencing - each lot follows a sequence of recipes in the wet bench area depending on the process, and each recipe has a sequence of steps. 


\subsection{Simulation Model Inputs}

Input data collected for the simulation model include a sample of process mix, recipe sequencing and recipe times. A sample of a process mix that has 11 main products is presented in Table 1 for the base simulation scenario together with percentages for each product, cumulative percentages and number of stages. Besides this sample process mix, the simulation model includes a sample of 37 different recipes and recipe sequences which may vary for each product stage. Also, each process has a different number of stages as given in Table 1. Stages in this context represent the number of revisits into the wet bench tool. Further, lots have deterministic lot sizes and they are assumed to have 25 wafers. It is important to note that the data for process mix, recipes and recipe sequences are obtained from a real fab and adapted for the simulation model.

Table 1: Input part mix.

\begin{tabular}{llll}
\hline Part & Percent & CumPercent & Stages \\
\hline A & 0.1481 & 0.1481 & 11 \\
B & 0.0185 & 0.1667 & 11 \\
C & 0.0185 & 0.1852 & 8 \\
D & 0.2222 & 0.4074 & 10 \\
E & 0.2593 & 0.6667 & 11 \\
F & 0.0185 & 0.6852 & 4 \\
G & 0.0185 & 0.7037 & 4 \\
H & 0.0556 & 0.7593 & 10 \\
I & 0.1481 & 0.9074 & 9 \\
K & 0.0556 & 0.963 & 11 \\
L & 0.037 & 1 & 7 \\
\hline
\end{tabular}

The lot releases are made according to an empirical distribution which shows the interarrival frequencies of the lots. Since the fab data do not fit any distributions, an empirical distribution is applied and interarrival frequencies of the lots are presented in Figure 2.

Histogram with 66 classes

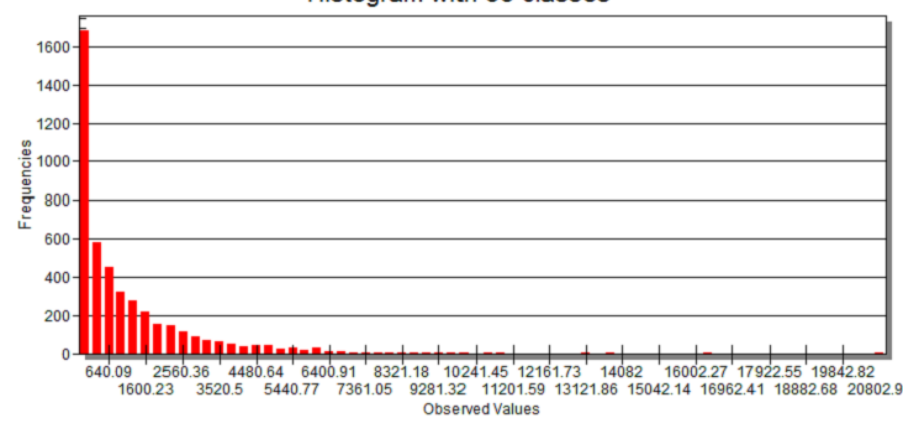

Figure 2: Interarrival frequencies of the lots.

In addition, priority levels of the lots are selected from an empirical distribution stochastically. Five levels of lot priorities are given in Table 2 in accordance to real system, which is the baseline priority levels for the lots represented as Set1. The lots with the first priority level have the highest presedence over the lots with the other priority levels. The lots with the fifth priority level which have the highest percentage in the simulation model have the lowest precedence. The other sets of priority levels used for simulation experiments are given in Table 7 in Appendix A.

The other stochastic elements of the wafer release mechanism includes, selection of process type and selection of stage and associated recipe. These stochastic elements are randomly selected from a uniform distribution. After the lots arrive at the wet bench queue, they are sorted according to the priority levels and then according to arrival times. In other words, a first-in first-out (FIFO) queue discipline is employed, which is the same queueing discipline as the real system has. Each recipe has a particular recipe time which is the time the carrier stays in the tank at a defined step. In addition, two different safety levels are defined for etch and rinse tanks in order to satisfy operational constraints. The etch tanks are assigned as critical to prevent overprocessing and scrapping, whereas rinse tanks are 
Table 2: Baseline priority levels (Set1).

\begin{tabular}{ll}
\hline Priority level & Percent $(\%)$ \\
\hline 0 & 2.17 \\
1 & 5.28 \\
2 & 1.57 \\
3 & 5.31 \\
4 & 85.67 \\
\hline
\end{tabular}

assigned as safe to allow infinite overprocessing. The base simulation model assumes three carriers, as in the real system. A carrier is used to load the lots having the same recipes in the wet bench process. The carrier capacity is assumed as two lots, or 50 wafers.

Apart from the above inputs, there are additional inputs related with robot operation which include robot speed, robot arms cleaning time, distance between each tank, robot ascending and descending time from a clearance height to the bottom of a tank. For the base simulation model, the robot speed is assumed as constant and $100 \mathrm{~mm} / \mathrm{sec}$, the robot arms cleaning time is $1 \mathrm{~min}$, the robot ascending and descending times are assumed as 3 seconds. In the simulation model, the distances between each tank are inserted from a real layout of a wet bench tool obtained from the wafer fab. Further, the transfer times from one particular tank to another one are calculated using these tank distances at the initilization stage of the model together with the inputs for the robot speed, the robot ascending and descending times. Therefore, the model shows the actual tool case in the fab.

A recipe sequence for a particular recipe specificies the steps that a batch follows in the wet bench tool. In the sample fab data, the recipe sequences of all of the recipes are categorized into seven different types according to the baths in which jobs are processed. These categorized recipe sequences are defined in Figure 3 for six etch tanks and six rinse tanks apart from dry and transfer modules.

\begin{tabular}{|c|c|c|c|c|c|c|c|c|c|c|c|c|c|c|c|}
\hline \multirow{2}{*}{$\begin{array}{c}\text { Recipe Sequence } \\
\text { Type No }\end{array}$} & \multicolumn{15}{|c|}{ Recipe Sequence (Bath Sequence) } \\
\hline & $\mathrm{T}$ & E1 & R1 & E2 & R2 & E3 & R3 & E4 & R4 & E5 & R5 & E6 & R6 & D & $\mathbf{T}$ \\
\hline Type1 & $\bar{x}$ & & & & & & & & & & & $\mathrm{x}$ & $x$ & $\bar{x}$ & $x$ \\
\hline Type2 & $x$ & $x$ & $x$ & $x$ & $x$ & $x$ & $x$ & & & & & & & $x$ & $x$ \\
\hline Type3 & $\mathrm{x}$ & & & & & $x$ & $x$ & & & & & & & $\mathrm{x}$ & $x$ \\
\hline Type4 & $x$ & & & & & & & $x$ & $x$ & & & & & $x$ & $x$ \\
\hline Type5 & $x$ & & & & & & & & & $x$ & $x$ & & & $x$ & $x$ \\
\hline Type6 & $\mathrm{x}$ & $x$ & $\mathrm{x}$ & & & $x$ & $\mathrm{X}$ & & & & & & & $x$ & $x$ \\
\hline Type7 & $x$ & $x$ & $x$ & $\mathrm{x}$ & $x$ & & & & & & & & & $x$ & $x$ \\
\hline
\end{tabular}

Figure 3: Categorized recipe sequences.

Table 3: Percentages of the recipes in the fab data.

\begin{tabular}{ll}
\hline Recipe Sequence & Percentage \\
\hline Type1 & 53.13 \\
Type2 & 11.46 \\
Type3 & 15.63 \\
Type4 & 4.17 \\
Type5 & 12.50 \\
Type6 & 1.04 \\
Type7 & 2.08 \\
\hline
\end{tabular}

To illustrate using the type1 recipe sequence, the first step is the transfer module, then the batch is moved into the etch6 (i.e. E6) tank followed by rinse6 (i.e R6), after which it is moved into the dry module (i.e. D) and finally the transfer module (i.e T). Moreover, a number of recipes in the sample fab data use the same recipe sequence. The summary of the percentage of recipes using each type of recipe sequence is given in the Table 3. According to Table 3,53.13\% of the total recipes used for all of the process stages have recipe sequences of type1, similarly $11.46 \%$ of the total recipes have type $2,15.63 \%$ of them have type $3,4.17 \%$ of them have type $4,12.50 \%$ of them have type $5,1.04 \%$ of them have type 6 and $2.08 \%$ of them have type 7 recipe sequences. 


\subsection{Simulation Model Outputs}

The wet bench simulation model was ran with a run length of 9 weeks (i.e 63 days). An initial bias of 6 days was removed to allow the system to reach steady state. Outputs of the simulation model are analyzed using three main performance measures at tool level, these performance measures are: average hourly throughput (i.e avg TH/hr), average cycle time (i.e avg CT) and average wait time (i.e avg WT). Since the objective of this study is to minimize the makespan, average cycle time is taken as main performance measure.

\subsection{Validation of Simulation Model}

The simulation model was validated using a number of methods, these methods are: (1) comparison of the tool operation especially for robot operations against the tool log data obtained from fab, (2) examining the animation and operation of the tool by an experienced engineer, (3) comparison of the results obtained from the simulation model with the results provided by the fab. Comparison of the simulation results with the fab data shows around $18 \%$ difference in weekly throughput results. However, it is important to note that simulation model assumes $100 \%$ availability, no chemical changeouts, no preventive maintenance and no random failures. If we take into account that approximately $85 \%$ of real tool availability which is provided by the fab, then the simulation results represent a maximum $5 \%$ of deviation from the real fab results. This is validated by also analyzing average daily throughput results.

\section{SIMULATION EXPERIMENTS AND RESULTS}

Simulation experiments are performed in order to examine the following factors which are considered to have an impact on the performance of wet bench tool in the real fab: robot speed, weekly wafer loading (start volume), priority lots and dry time. Each factor is assumed to have three levels which creates $3^{4}=81$ design points. The factors and their levels are shown in Table 4 and a summary of the simulation results are given in Table 5. Analysis of variance (ANOVA) is performed for screening purposes to understand the impact of the factors on the tool performance. Figure 4 presents the effects of the main factors in the ANOVA.

Table 4: Experimental design.

\begin{tabular}{l|lll}
\hline Factor & Low & Medium & High \\
\hline Weekly wafer loading & 1350 wafers/week & 1500 wafers/week & 1650 wafer/week \\
Dry time & $10 \mathrm{~min}$ & $12 \mathrm{~min}$ & $14 \mathrm{~min}$ \\
Robot speed & $100 \mathrm{~mm} / \mathrm{s}$ & $110 \mathrm{~mm} / \mathrm{s}$ & $120 \mathrm{~mm} / \mathrm{s}$ \\
Priority lots & Set1 & Set2 & Set3 \\
\hline
\end{tabular}

Table 5: Summary of the simulation results.

\begin{tabular}{l|lll}
\hline Factor & Avg TH/hr & Avg CT & Avg WT \\
\hline Weekly wafer loading & $\Uparrow$ & $\Uparrow$ & $\Uparrow$ \\
Dry time & $\Downarrow$ & $\Uparrow$ & $\Uparrow$ \\
Priority lots & $\Downarrow$ very little & $\Uparrow$ & $\Uparrow$ \\
Robot speed & $\Uparrow$ very little & $\Downarrow$ to some point & $\Downarrow$ \\
\hline
\end{tabular}

According to the ANOVA results, the increase in weekly loading levels and dryer time are the most significant factors on the average cycle time. In other words, these factors drive up the average cycle time significantly in large amounts. On the other hand, the increase in priority lots increase the average cycle time in small amounts. However, the increase in robot speed decrease the average cycle time to a certain point, after that no significant decrease is observed in average cycle time. Further, simulation experiments are performed under increasing weekly loadings with five replications. Each average cycle time (avg CT) and average throughput per hour (avg TH/hr) results are paired in Figure 5. Also, a performance curve is drawn for the results.

Additional simulation experiments are performed to improve the wet bench capacity by the reconfiguration of recipe sequences. The reconfiguration of the wet bench tool is needed due to 


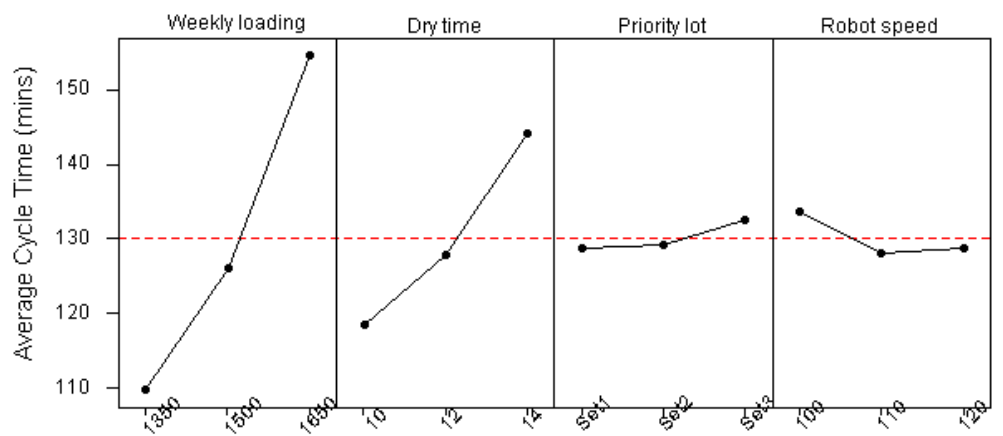

Figure 4: Main effects ANOVA plot for average cycle time.

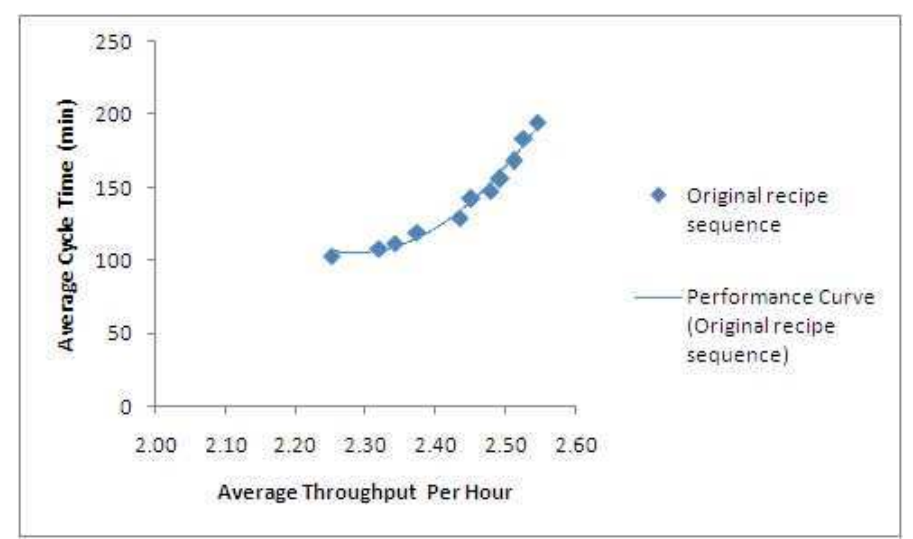

Figure 5: Results of simulation experiments with original recipe sequences.

process changes, or process migration or quality reasons in the tool, and it may take significant engineering time due to the qualification of the bath processes and recipes. Simulation experiments are performed to analyze the impact of changes in recipe sequences on the tool capacity. To do this, the steps for the third etch tank (i.e. E3) and the third rinse tank (i.e. R3) are removed from type 2 recipe sequence due to the process requirements for the recipes using type 2 sequence. The second etch bath time for the recipes using type 2 sequence is very high. Therefore, the need for the third etch bath together with its rinse bath is eliminated by process engineers for both process and improvement reasons. In other words, type 2 recipe sequence is merged with type 7 recipe sequence by removing the third etch and rinse baths, and the percentage of recipes using type 7 recipe sequence is increased as given in Table 6.

Table 6: Modified percentages of the recipes in the fab data.

\begin{tabular}{ll}
\hline Recipe Sequence & Percentage \\
\hline Type1 & 53.13 \\
Type3 & 15.63 \\
Type4 & 4.17 \\
Type5 & 12.50 \\
Type6 & 1.04 \\
Type7 & 13.44 \\
\hline
\end{tabular}

In addition, the moves and loadings of the third etch and rinse tanks are decreased since they are removed from recipe sequences of type 2 . Figure 6 shows the modified recipe sequences. 
Kabak, Heavey and Corbett

\begin{tabular}{|c|c|c|c|c|c|c|c|c|c|c|c|c|c|c|c|}
\hline \multirow{2}{*}{$\begin{array}{c}\text { Recipe Sequence } \\
\text { Type No }\end{array}$} & \multicolumn{15}{|c|}{ Recipe Sequence (Bath Sequence) } \\
\hline & $\mathrm{T}$ & E1 & R1 & E2 & R2 & E3 & R3 & E4 & R4 & E5 & R5 & E6 & R6 & D & $T$ \\
\hline Type1 & $x$ & & & & & & & & & & & $x$ & $x$ & $\bar{x}$ & $\bar{x}$ \\
\hline Type3 & $x$ & & & & & $x$ & $x$ & & & & & & & $x$ & $x$ \\
\hline Type4 & $x$ & & & & & & & $x$ & $x$ & & & & & $x$ & $x$ \\
\hline Type5 & $x$ & & & & & & & & & $X$ & $x$ & & & $x$ & $x$ \\
\hline Type6 & $x$ & $x$ & $x$ & & & $x$ & $x$ & & & & & & & $x$ & $x$ \\
\hline Type7 & $x$ & $x$ & $x$ & $X$ & $X$ & & & & & & & & & $x$ & $x$ \\
\hline
\end{tabular}

Figure 6: Reconfiguration of recipe sequences.

To analyze the impact of recipe sequence changes on the tool performance, the simulation experiments with the modified recipe sequencess are compared with the original recipe sequences. The results with original recipe sequences are compared in Figure 7. According to the results, the average hourly throughput is increased by $18 \%$ under the same average cycle time. The results show that wet bench capability is significantly improved by reconfiguration of recipe sequences.

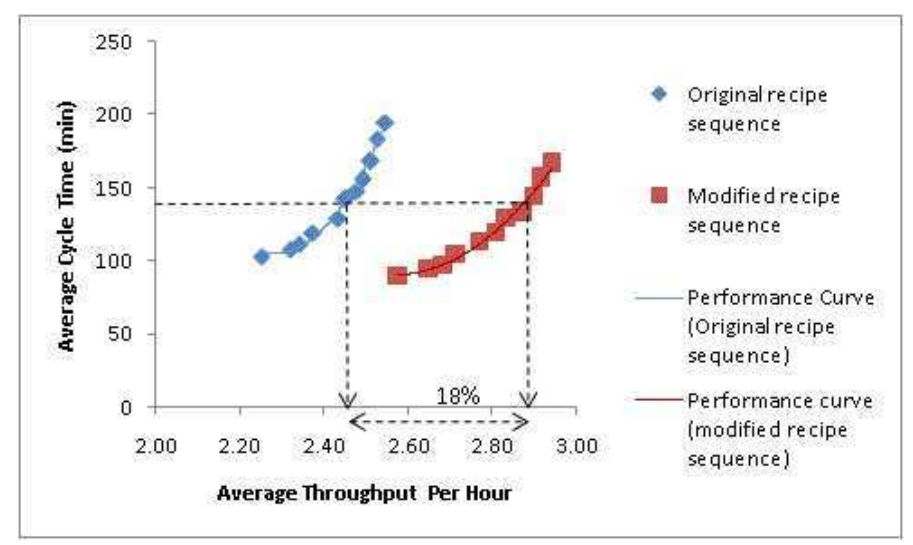

Figure 7: Results of Simulation experiments under modified recipe sequences.

\section{CONCLUDING REMARKS}

A detailed simulation model is built to understand the impact of operational factors on the wet bench performance. Using the significance of the results, further simulation experiments are conducted to improve the wet bench performance. The results show that wet bench capability is significantly improved by reconfiguration of recipe sequences. The best way to optimize the wet bench was found to be reducing the recipe time of the longer recipe sequence (i.e reducing the etch and rinse time). After that the rationalization of similar recipes to reduce the number of distinct recipes was the next most important action. Also, the reduction in dry time yielded significant improvements in wet bench capability in the experimental design. Where a step increase in capacity was needed, the model provided quantitative information regarding to the benefit of removing a recipe from the bench.

\section{A APPENDICES}

Table 7: Priority levels in experimental design (Set2 and Set3).

\begin{tabular}{lll}
\hline Priority level & Set2 $(\%)$ & Set3 $(\%)$ \\
\hline 0 & 4.34 & 8.68 \\
1 & 10.56 & 21.12 \\
2 & 3.14 & 6.28 \\
3 & 10.62 & 21.24 \\
4 & 71.34 & 42.68 \\
\hline
\end{tabular}




\section{REFERENCES}

Bhushan, S., and I. A. Karimi. 2003. An milp approach to automated wet-etch station scheduling. Industrial \& Engineering Chemistry Research 42 (7): 1391-1399.

Geiger, C. D., K. G. Kempf, and R. Uzsoy. 1997. A tabu search approach to scheduling an automated wet etch station. Journal of Manufacturing Systems 16 (2): 102-116.

Govind, N., and D. Fronckowiak. 2003. Resident-entity based simulation of batch chamber tools in $300 \mathrm{~mm}$ semiconductor manufacturing. In Winter Simulation Conference, Volume 2, 1398-1405 vol.2.

Lee, T.-E., H.-Y. Lee, and S.-J. Lee. 2007. Scheduling a wet station for wafer cleaning with multiple job flows and multiple wafer-handling robots. International Journal of Production Research 45 (3): $487-507$.

Mauer, J. L., R. E. A. Schelasin, and P. J. Miller. 1992. Analysis of cluster tool performance in semiconductor manufacturing. In Thirteenth IEEE/CHMT International Electronics Manufacturing Technology Symposium, 129-134.

Quek, P. T., G. Boon Ping, T. Song Lian, P. Chan Lai, and B. vd Heijden. 2007. Analysis of the front-end wet strip efficiency performance for productivity. In ISSM International Symposium on Semiconductor Manufacturing, 1-4.

Siemens 2008. Tecnomatix plant simulation $\quad$ software $\quad 8.2$. <http://www.plm. automation. siemens. coms.

\section{AUTHOR BIOGRAPHIES}

KAMIL ERKAN KABAK is currently pursuing the Ph.D. degree in the Manufacturing and Operations Engineering Department at the University of Limerick, Ireland. He received his bachelor degree in the Department of Environmental Engineering from Middle East Technical University, Turkey. Later, he obtained his master degree from the Department of Industrial Engineering, Dokuz Eylul University, Turkey. His research interests include instability of production plans, simulation modeling along with its applications to analysis and control methods for semiconductor manufacturing. He is a member of INFORMS and Operational Research Society of Turkey. He can be reached via email at <erkan.kabak@ul.ie>.

CATHAL HEAVEY is a Senior Lecturer of Operations Management in the Department of Manufacturing and Operations Engineering at the University of Limerick. He is an Industrial Engineering graduate of the National University of Ireland (University College Galway) and holds a M. Eng.Sc. and $\mathrm{Ph}$.D. from the same University. He has published in the areas of queuing and simulation modelling. Research interests are: Simulation Modelling of Discrete Event Systems; Modelling and Analysis of Supply Chains and Manufacturing Systems; Process modelling; Component- based simulation; Decision support systems. His email address is <cathal.heavey@ul.ie $>$.

VINCENT CORBETT is a Senior Manufacturing Engineer with Analog Devices in Limerick Ireland. He is a Laboratory Science Graduate of Limerick Institute of Technology Ireland and holds a MBA from Oxford Brookes University in the UK. His email address is <vincent. corbett@analog. com>. 